High Energy Phenomena in Relativistic Outflows III (HEPRO III)

International Journal of Modern Physics: Conference Series

Vol. 8 (2012) 144-150

(C) World Scientific Publishing Company

DOI: $10.1142 / \mathrm{S} 2010194512004539$

\title{
PARTICLE ACCELERATION AT THE TERMINATION SHOCK OF STRIPED PULSAR WINDS
}

\author{
LORENZO SIRONI* and ANATOLY SPITKOVSKY ${ }^{\dagger}$ \\ Department of Astrophysical Sciences, Princeton University, 4 Ivy Lane \\ Princeton, NJ 08544-1001, USA \\ *lsironi@astro.princeton.edu; †anatoly@astro.princeton.edu
}

\begin{abstract}
The relativistic wind of pulsars consists of toroidal stripes of opposite magnetic field polarity, separated by current sheets of hot plasma. By means of multi-dimensional particle-in-cell simulations, we investigate particle acceleration and magnetic field dissipation at the termination shock of a relativistic striped pulsar wind. At the shock, the flow compresses and the alternating fields annihilate by driven magnetic reconnection. Irrespective of the stripe wavelength $\lambda$ or the wind magnetization $\sigma$ (in the regime $\sigma \gg 1$ of magnetically dominated flows), shock-driven reconnection transfers all the magnetic energy of alternating fields to the particles. In the limit $\lambda /\left(r_{L} \sigma\right) \gg 1$, where $r_{L}$ is the relativistic Larmor radius in the wind, the post-shock spectrum approaches a flat powerlaw tail with slope around -1.5 , populated by particles accelerated by the reconnection electric field. Our findings place important constraints on the models of non-thermal radiation from Pulsar Wind Nebulae.
\end{abstract}

Keywords: Acceleration of particles; pulsars: general; shock waves

\section{Introduction}

The broadband spectrum of Pulsar Wind Nebulae (PWNe) is usually modeled as synchrotron and inverse Compton radiation from a nonthermal population of electron-positron pairs (hereafter, simply "electrons"), presumably accelerated at the termination shock of the relativistic pulsar wind. The observed radio spectrum $\left(F_{\nu} \propto \nu^{-0.25}\right.$ for the Crab Nebula $\left.{ }^{1}\right)$ implies a distribution of emitting electrons of the form $d N / d E \propto E^{-p}$, with a flat slope $p \sim 1.5$. To explain the radio through optical emission of the Crab, the power law of shock-accelerated electrons should span at least three decades in particle energy.

A flat power-law spectrum with index $1<p<2$ is not expected from Fermi acceleration in relativistic shocks, which normally yields slopes $p>2 .^{2,3,4,5}$ An acceleration mechanism capable of generating flat spectra in PWNe was discussed by Ref. 6 , under the assumption that the flow upstream of the termination shock consists of alternating stripes of opposite magnetic polarity, separated by current sheets of hot plasma (from now on, a "striped wind"). For obliquely-rotating pulsars, this is the configuration expected around the equatorial plane of the wind, where the sign of the toroidal field alternates with the pulsar period. 
We explore via multi-dimensional fully-kinetic particle-in-cell (PIC) simulations the acceleration of particles at the termination shock of a striped relativistic electron-positron wind. We find that the alternating fields are completely dissipated upon compression by the shock, via a process known as "driven magnetic reconnection." All the energy in alternating fields is transferred to the particles, regardless of the properties of the flow. Broad particle spectra with flat slopes $(1<p<2)$ are a common by-product of shock-driven reconnection, but we show that the extent of the power-law tail depends on the wind magnetization and the stripe wavelength.

\section{Simulation Setup}

We use the 3D electromagnetic PIC code TRISTAN-MP. ${ }^{7,8}$ We mainly utilize 2D computational domains in the $x y$ plane, with periodic boundary conditions in the $y$ direction. However, we have verified in Ref. 9 that our 2D runs capture most of the relevant $3 \mathrm{D}$ physics. The incoming electron-positron stream is injected along $-\hat{\boldsymbol{x}}$ with bulk Lorentz factor $\gamma_{0}=15$ (but we have explored a wide range of $\gamma_{0}$, finding the same results, modulo an overall shift in the energy scale ${ }^{9}$ ). The shock moves toward $+\hat{\boldsymbol{x}}$, and the simulation is performed in the post-shock rest frame. The upstream flow carries a strong magnetic field of intensity $B_{0}$, oriented along the $y$ direction, alternating with wavelength $\lambda$. We vary $\lambda$ from $\lambda=20 \mathrm{c} / \omega_{\mathrm{p}}$ up to $\lambda=1280 c / \omega_{\mathrm{p}}$, where $c / \omega_{\mathrm{p}} \equiv \sqrt{\gamma_{0} m c^{2} / 4 \pi e^{2} n_{c 0}}$ is the relativistic plasma skin depth in the wind. Here, $n_{c 0}$ is the density of particles in the wind. The thickness of the current sheets is a few plasma skin depths (so that the process of "undriven reconnection" is suppressed in our simulations), but our results are not sensitive to this parameter, provided that the contribution of current sheets to the particle and energy flux in the wind is negligible. We parameterize the field strength $B_{0}$ via the so-called magnetization parameter $\sigma \equiv B_{0}^{2} / 4 \pi \gamma_{0} m n_{c 0} c^{2}$. We vary $\sigma$ from 10 up to 100. Although the magnetic field strength in the wind is always $B_{0}$, the wavelength-averaged field $\left\langle B_{y}\right\rangle_{\lambda}$ can vary from zero up to $B_{0}$, depending on the relative widths of the regions of positive and negative field. In pulsar winds, one expects $\left\langle B_{y}\right\rangle_{\lambda}=0$ only in the equatorial plane (where the stripes are symmetric), whereas $\left|\left\langle B_{y}\right\rangle_{\lambda}\right| / B_{0} \rightarrow 1$ at high latitudes. We choose $\alpha=2\left\langle B_{y}\right\rangle_{\lambda} /\left(B_{0}+\left|\left\langle B_{y}\right\rangle_{\lambda}\right|\right)=$ 0.1 (corresponding to $\left\langle B_{y}\right\rangle_{\lambda} / B_{0} \simeq 0.05$ ), to represent the behavior of the shock in the vicinity of the equatorial plane. In Ref. 9 we explore the full range $0 \leq \alpha \leq 1$.

We resolve the smallest scale in the system, which for $\sigma>1$ is the relativistic Larmor radius $r_{L}=\left(c / \omega_{\mathrm{p}}\right) / \sqrt{\sigma}$, with a few computational cells. Our computational domain is typically $\sim 400 \mathrm{c} / \omega_{\mathrm{p}}$ wide (along the $y$ direction). As we comment below, a large box is of paramount importance for the consistency of our findings.

\section{Shock Structure and Particle Acceleration}

Fig. 1 shows the typical structure of a relativistic shock propagating in a striped wind with $\lambda=640 \mathrm{c} / \omega_{\mathrm{p}}, \sigma=10$, and $\alpha=0.1$. In $\S 4$ we discuss the dependence of our results on the stripe wavelength and the magnetization (see also Ref. 9). 
The steady-state structure of the flow in Fig. 1 shows the presence of two shocks. The main shock (which we shall call the "hydrodynamic shock" from now on, for reasons that will become clear below) corresponds to the transition at $x \simeq 1000 \mathrm{c} / \omega_{\mathrm{p}}$. At $x \simeq 2600 c / \omega_{\mathrm{p}}$, well ahead of the hydrodynamic shock, the incoming flow crosses a fast magnetohydrodynamic (MHD) shock. The compression induced by the passage of the fast shock through a given current sheet triggers the onset of magnetic reconnection. Small-scale islands develop inside the current sheet as a result of the tearing mode instability, and they grow and coalesce as the flow recedes from the fast shock (see the 2D plots of density and magnetic energy of Fig. 1(a) and (b), respectively). A magnetic X-point exists in between each pair of neighboring islands, where field lines of opposite polarity break and reconnect. Particles flow into the $\mathrm{X}$-points and are accelerated by the reconnection electric field (see the 2D plot of $\epsilon_{B}-\epsilon_{E}$ in Fig. 1(c)), while being advected by the reconnected magnetic field into the closest island. Magnetic islands act as reservoirs of particles (Fig. 1(a)) and
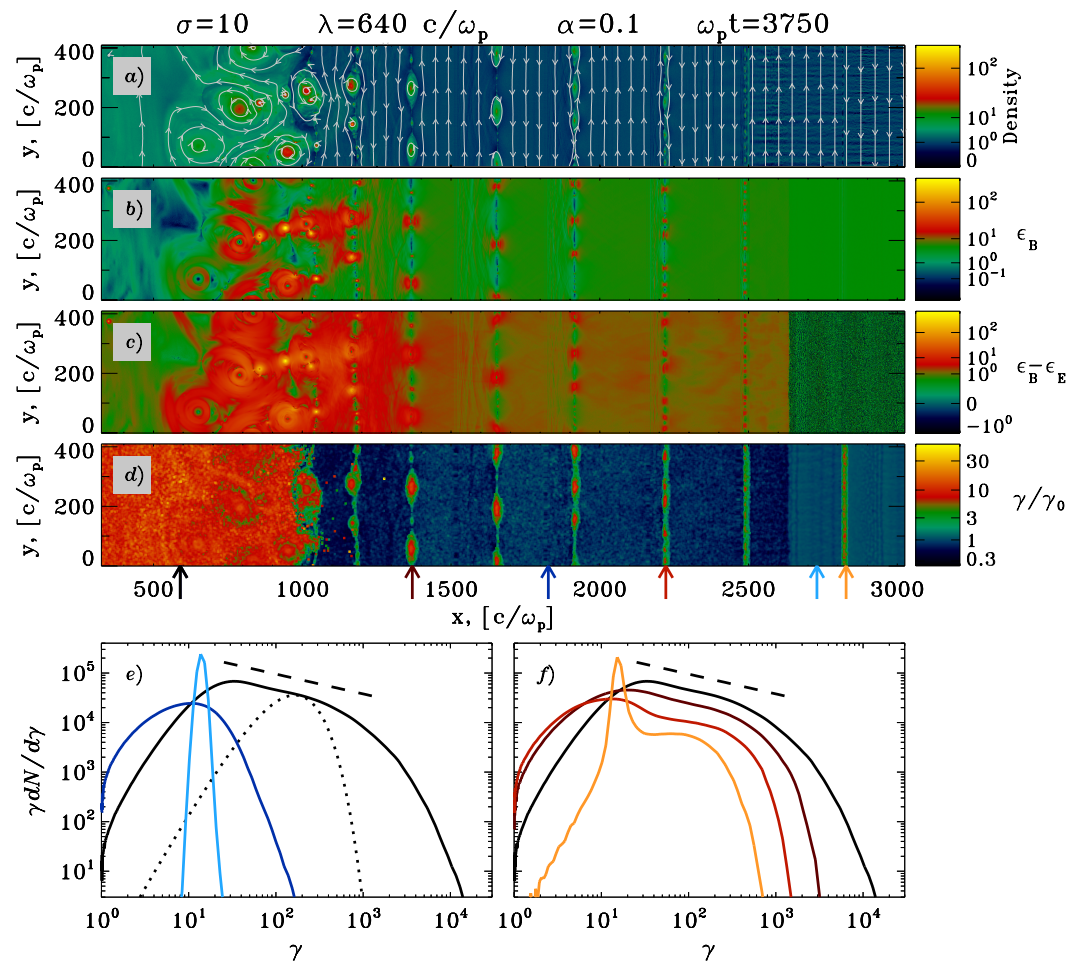

Fig. 1. Internal structure of the flow, zooming in on a region around the shock. We show the 2D plots of: (a) particle number density, with contours showing the magnetic field lines; (b) magnetic energy fraction $\epsilon_{B} \equiv B^{2} / 8 \pi \gamma_{0} m n_{c 0} c^{2} ;$ (c) $\epsilon_{B}-\epsilon_{E}$, where $\epsilon_{E} \equiv E^{2} / 8 \pi \gamma_{0} m n_{c 0} c^{2} ;$ (d) mean kinetic energy per particle. Panels (e) and (f) show the particle energy spectra, respectively outside (e) or inside (f) of current sheets. The color of each spectrum matches the color of the corresponding arrow at the bottom of panel (d), showing where the spectrum is computed. The dotted line in panel (e) is a Maxwellian with the same average energy as the downstream particles; the dashed lines in panels (e) and (f) indicate a power law with slope $p=1.4$. 
particle energy (the high-energy regions of panel (d), colored in red, match very well the overdense islands of panel (a)).

As reconnection proceeds downstream from the fast shock, more and more magnetic energy is released and transferred to particles, until magnetic islands fill the entire region between neighboring current sheets. Now, the striped structure of the flow is erased, and a hydrodynamic shock forms (located at $x \simeq 1000 c / \omega_{\mathrm{p}}$ in Fig. 1). The jump conditions across the shock are appropriate for a relativistic unmagnetized 3D plasma. Behind the shock, the only residual magnetic field comes from shock-compression of the stripe-averaged field $\left\langle B_{y}\right\rangle_{\lambda}$. As the flow propagates from the fast to the hydrodynamic shock, the particles accelerated by the reconnection electric field populate a prominent high-energy component in the particle spectra within current sheets (Fig. 1(f), yellow through black lines). Downstream from the hydrodynamic shock, the particle spectrum (black line in Fig. 1(e) and (f)) is in the form of a flat power-law tail (with spectral index $p \simeq 1.4$, dashed line), much broader than a Maxwellian with the same mean energy (dotted line in Fig. 1(e)).

Finally, we point out that, as apparent in Fig. 1, the tearing mode instability, which plays an essential role for the dynamics of the flow, can be captured correctly only with multi-dimensional simulations. Indeed, for the parameters employed in this section, the 1D model by Ref. 10 would predict negligible field dissipation, in sharp contrast with our findings. This clearly emphasizes the importance of multidimensional physics for our understanding of shock-driven reconnection.

\section{Dependence on the Wind Properties}

In this section, we show that complete field dissipation (and transfer of field energy to the particles) occurs irrespective of the wind properties, but the width of the downstream particle spectrum is sensitive to the stripe wavelength and the wind magnetization. Fig. 2 shows how the downstream particle spectrum changes for different stripe wavelengths $\lambda$, keeping fixed the magnetization $\sigma=10$ and the stripe-averaged field $\left\langle B_{y}\right\rangle_{\lambda} / B_{0} \simeq 0.05$ (corresponding to $\alpha=0.1$ ). In all cases, the value of the post-shock mean particle Lorentz factor $\langle\gamma\rangle$ measured in our simulations (black line in the subpanel of Fig. 2) is consistent with full dissipation of the alternating fields, yielding $\langle\gamma\rangle \simeq \gamma_{0} \sigma$. Even though the mean kinetic energy per particle does not appreciably vary with $\lambda$, the shape of the spectrum does change, with a clear tendency for broader spectra at longer stripe wavelengths.

This trend can be easily understood by considering the structure of the flow, just upstream of the hydrodynamic shock. Here, the size of reconnection islands is constrained by the distance between neighboring current sheets, which is proportional to $\lambda$. Shorter wavelengths will then result in more numerous islands of smaller size, whereas fewer but bigger islands will be present for longer $\lambda$ (see Fig. 1). Since an $\mathrm{X}$-point exists in between each pair of neighboring islands (belonging to the same current sheet), the number of X-points per unit length (along the current sheet) will be larger for smaller $\lambda$. For short wavelengths, most of the incoming particles will likely pass in the vicinity of one of the numerous X-points, thus gaining energy 
from the reconnection electric field. The energy evolution of one particle will then be similar to that of any other particle, with its Lorentz factor increasing from $\gamma \simeq \gamma_{0}$ up to $\gamma \simeq \gamma_{0} \sigma$, which results in a narrow Maxwellian-like distribution. On the other hand, for long wavelengths the energy evolution of different particles can be extremely diverse, depending on how far they pass from the closest X-point. Most of the particles stay far from X-points and remain cold, but the particles that interact with an X-point are accelerated to high energies by the reconnection electric field. This results in a broad energy spectrum.

We find that the threshold between short and long wavelengths depends on the stripe wavelength and the wind magnetization via the combination $\lambda /\left(r_{L} \sigma\right)$, namely the stripe wavelength measured in units of the post-shock plasma skin depth. In Fig. 3 we confirm that the main properties of the shock, and in particular the high-energy end of the downstream particle spectrum, are relatively insensitive to variations in $\lambda$ or $\sigma$, provided that the ratio $\lambda /\left(r_{L} \sigma\right)$ is kept constant. When varying $\lambda /\left(r_{L} \sigma\right)$, a Maxwellian-like spectrum is obtained for $\lambda /\left(r_{L} \sigma\right) \lesssim$ a few tens, whereas in the limit $\lambda /\left(r_{L} \sigma\right) \gg 1$ the spectrum approaches a broad power-law tail of index $1<p<2$, extending from $\gamma_{\min } \simeq \gamma_{0}$ up to $\gamma_{\max } \simeq \gamma_{0} \sigma^{1 /(2-p)}$.

Finally, we remark that the minor bump emerging at high energies in the spectra of Figs. 2 and 3 is populated by particles that are accelerated via the shock-drift

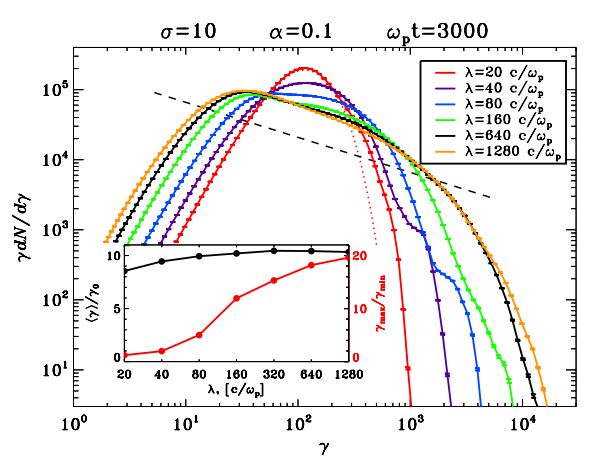

Fig. 2. Downstream particle spectrum for different values of $\lambda$, in a flow with $\sigma=10$ and $\alpha=0.1$. The dotted red line is a Maxwellian with the same average energy as the spectrum colored in red (which refers to $\lambda=20 \mathrm{c} / \omega_{\mathrm{p}}$ ); the dashed black line is a power law with slope $p=1.5$. In the subpanel, the black line shows the average Lorentz factor of downstream particles $\langle\gamma\rangle$ as a function of $\lambda$ (axis on the left), whereas the red line presents the dependence of $\gamma_{\max } / \gamma_{\min }$ on $\lambda$ (axis on the right). Here, $\gamma_{\min }$ is the location where $\gamma d N / d \gamma$ peaks (i.e., where most of the particles reside), whereas $\gamma_{\max }$ is the Lorentz factor where $\gamma^{2} d N / d \gamma$ peaks (i.e., where most of the energy lies).

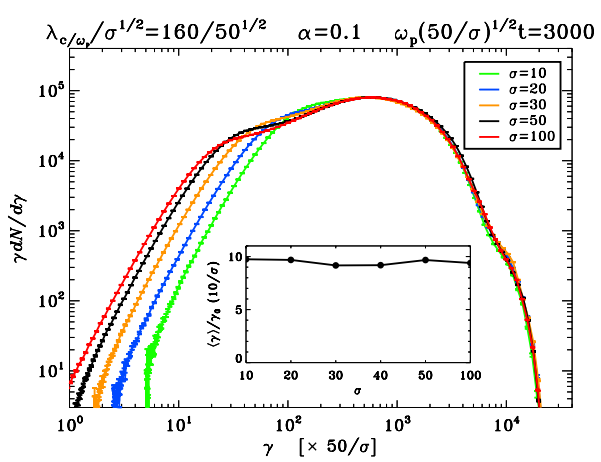

Fig. 3. Downstream particle spectrum for different values of $\lambda$ and $\sigma$, but such that the ratio $\lambda /\left(r_{L} \sigma\right) \simeq 22.5$ is kept fixed (as well as $\alpha=0.1)$. Here, $\lambda /\left(r_{L} \sigma\right)$ is roughly the wind wavelength in units of the post-shock plasma skin depth. The comparison is performed at the same time, in units of the relativistic plasma frequency of the post-shock flow $\left(\simeq \omega_{\mathrm{p}} / \sqrt{\sigma}\right)$. Spectra are shifted along the $x$-axis by $50 / \sigma$ to facilitate comparison with the case $\sigma=50$. The black line in the subpanel shows the average downstream Lorentz factor $\langle\gamma\rangle$ as a function of $\sigma$. 
mechanism ${ }^{11,12,13}$ at the hydrodynamic shock. They gain energy from the stripeaveraged motional electric field $\left\langle E_{z}\right\rangle_{\lambda} \simeq\left\langle B_{y}\right\rangle_{\lambda}$, while gyrating around the shock.

\section{Summary and Discussion}

We have explored by means of multi-dimensional PIC simulations the internal structure and acceleration properties of relativistic shocks that propagate in a striped pulsar wind, i.e., a flow consisting of stripes of alternating field polarity separated by current sheets of hot plasma. We find that, regardless of the stripe wavelength $\lambda$ or the wind magnetization $\sigma$, at the termination shock the energy stored in the alternating fields is entirely transferred to the particles via shock-driven magnetic reconnection, and the downstream fluid behaves like an unmagnetized plasma. The shape of the post-shock spectrum depends primarily on the combination $\lambda /\left(r_{L} \sigma\right)$, where $r_{L}$ is the relativistic Larmor radius in the striped wind. As $\lambda /\left(r_{L} \sigma\right)$ increases, the spectrum passes from a narrow Maxwellian-like distribution to a broad powerlaw tail of index $1<p<2$, whose extent is $\gamma_{\max } / \gamma_{\min } \simeq \sigma^{1 /(2-p)}$.

The particle energy spectra extracted from our simulations can be used directly to interpret the radiative signature of PWNe. The radio spectrum of the Crab Nebula, the prototype of the class of PWNe, requires a population of nonthermal particles with a flat spectral slope $(p \simeq 1.5)$, extending from $10^{2} \mathrm{MeV}$ up to $10^{5} \mathrm{MeV}$. Our results suggest that, for $p \simeq 1.5$, the particle spectrum will extend over three decades in energy if the wind magnetization at the termination shock is $\sigma \gtrsim 30$. In addition, broad particle spectra are generated only if $\lambda /\left(r_{L} \sigma\right) \gtrsim$ a few tens. At the termination shock of the pulsar wind $\left(R=R_{T S}\right)$ we have

$$
\frac{\lambda}{r_{L} \sigma} \simeq 4 \pi \kappa \frac{R_{L C}}{R_{T S}}
$$

where $R_{L C}=c / \Omega$ is the light cylinder radius ( $\Omega$ is the pulsar rotational frequency), and $\kappa$ is the so-called multiplicity in the wind (i.e., the ratio of the actual density to the Goldreich-Julian density $\left.{ }^{14}\right)$. For the Crab, $R_{T S} \simeq 5 \times 10^{8} R_{L C}{ }^{15}$ and most available models estimate $\kappa \simeq 10^{4}-10^{6} .{ }^{16}$ Based on our findings, the resulting value of $\lambda /\left(r_{L} \sigma\right) \lesssim 0.01$ would yield a Maxwellian-like spectrum, at odds with the wide flat spectrum required by observations. If radio-emitting electrons are produced in the equatorial plane by shock-driven reconnection, a revision of the existing estimates of $\kappa$ is required. In this respect, we point out that the values of $\kappa$ quoted above are averages over latitude, and one cannot exclude that particle injection into the pulsar wind is highly anisotropic, with multiplicity as large as $10^{8}$ along the equatorial plane. This is not in conflict with the observed lack of gradients in the radio spectral slope of the Crab, ${ }^{17}$ since strong fluid motions downstream from the termination shock would quickly fill the entire nebula with the long-lived radio-emitting electrons. ${ }^{18}$ Alternatively, the radio part of the spectrum may be produced at higher latitudes, where the termination shock is closer to the pulsar, and the ratio in eq. (1) becomes larger. In summary, our findings place important 
constraints on the magnetospheric physics of rotation-powered pulsars, and they provide physically-grounded inputs for models of nonthermal emission in PWNe.

\section{References}

1. M. F. Bietenholz et al., Astrophys. J. 490, 291 (1997).

2. A. Achterberg et al., Mon. Not. R. Astron. Soc. 328, 393 (2001).

3. U. Keshet and E. Waxman, Phys. Rev. Lett. 94, 111102 (2005).

4. L. Sironi and A. Spitkovsky, Astrophys. J. 698, 1523 (2009).

5. L. Sironi and A. Spitkovsky, Astrophys. J. 726, 75 (2011).

6. Y. E. Lyubarsky, Mon. Not. R. Astron. Soc. 345, 15 (2003).

7. O. Buneman, in Computer Space Plasma Physics (Terra Scientific, Tokyo, 1993), p. 67

8. A. Spitkovsky, in Astrophysical Sources of High Energy Particles and Radiation, ed. T. Bulik, B. Rudak, and G. Madejski (AIP Conf. Ser., 2005), p. 345

9. L. Sironi and A. Spitkovsky, Astrophys. J. 741, 39 (2011).

10. J. Pétri and Y. Lyubarsky, Astron. Astrophys. 473, 683 (2007).

11. G. Chen and T. P. Armstrong, in International Cosmic Ray Conference (1975), p. 1814.

12. G. M. Webb, W. I. Axford and T. Terasawa, Astrophys. J. 270, 537 (1983).

13. M. C. Begelman and J. G. Kirk, Astrophys. J. 353, 66 (1990).

14. P. Goldreich and W. H. Julian, Astrophys. J. 157, 869 (1969).

15. J. J. Hester et al., Astrophys. J. Lett. 577, L49 (2002).

16. N. Bucciantini, J. Arons and E. Amato, Mon. Not. R. Astron. Soc. 410, 381 (2011).

17. M. F. Bietenholz and P. P. Kronberg, Astrophys. J. 393, 206 (1992).

18. L. Del Zanna, E. Amato and N. Bucciantini, Astron. Astrophys. 421, 1063 (2004). 